\title{
Isoperimetric Problems of the Calculus of Variations on Time Scales
}

\author{
Rui A. C. Ferreira and Delfim F. M. Torres
}

\begin{abstract}
We prove a necessary optimality condition for isoperimetric problems on time scales in the space of delta-differentiable functions with rdcontinuous derivatives. The results are then applied to Sturm-Liouville eigenvalue problems on time scales.
\end{abstract}

\section{Introduction}

The theory of time scales (see Section 2 for basic definitions and results) is a relatively new area, that unify and generalize difference and differential equations 8. It was initiated by Stefan Hilger in the nineties of the XX century [12, 13, and is now subject of strong current research in many different fields in which dynamic processes can be described with discrete or continuous models [1].

The study of the calculus of variations on time scales has began in 2004 with the paper [6] of Bohner, where the necessary optimality conditions of Euler-Lagrange and Legendre, as well as a sufficient Jacobi-type condition, are proved for the basic problem of the calculus of variations with fixed endpoints. Since the pioneer paper [6], the following classical results of the calculus of variations on continuous-time $(\mathbb{T}=\mathbb{R})$ and discrete-time $(\mathbb{T}=\mathbb{Z})$ have been unified and generalized to a time scale $\mathbb{T}$ : the Noether's theorem [5]; the Euler-Lagrange equations for problems of the calculus of variations with double integrals [7] and for problems with higherorder derivatives 10; transversality conditions 14]. The more general theory of the calculus of variations on time scales seems to be useful in applications to Economics 4. Much remains to be done [1], and here we give a step further. Our main aim is to obtain a necessary optimality condition for isoperimetric problems on time scales. Corollaries include the classical case $(\mathbb{T}=\mathbb{R})$, which is extensively studied in the literature (see, e.g., [15); and discrete-time versions [3].

The plan of the paper is as follows. Section 2 gives a short introduction to time scales, providing the definitions and results needed in the sequel. In Section 3 we prove a necessary optimality condition for the isoperimetric problem on time scales

2000 Mathematics Subject Classification. 49K05, 39A12.

This is a preprint accepted (July 16, 2008) for publication in the Proceedings of the Conference on Nonlinear Analysis and Optimization, June 18-24, 2008, Technion, Haifa, Israel, to appear in Contemporary Mathematics. The first author was supported by the PhD fellowship SFRH/BD/39816/2007; the second author by the R\&D unit CEOC, via FCT and the EC fund FEDER/POCI 2010. 
(Theorem 3.4); then, we establish a connection (Theorem 3.7) with the previously studied Sturm-Liouville eigenvalue problems on time scales [2].

\section{The calculus on time scales and preliminaries}

We begin by recalling the main definitions and properties of time scales (cf. 1, 8, 12, 13. and references therein).

A nonempty closed subset of $\mathbb{R}$ is called a Time Scale and is denoted by $\mathbb{T}$. The forward jump operator $\sigma: \mathbb{T} \rightarrow \mathbb{T}$ is defined by $\sigma(t)=\inf \{s \in \mathbb{T}: s>t\}$, for all $t \in \mathbb{T}$, while the backward jump operator $\rho: \mathbb{T} \rightarrow \mathbb{T}$ is defined by $\rho(t)=$ $\sup \{s \in \mathbb{T}: s<t\}$, for all $t \in \mathbb{T}$, with $\inf \emptyset=\sup \mathbb{T}$ (i.e., $\sigma(M)=M$ if $\mathbb{T}$ has a maximum $M$ ) and $\sup \emptyset=\inf \mathbb{T}$ (i.e., $\rho(m)=m$ if $\mathbb{T}$ has a minimum $m$ ). A point $t \in \mathbb{T}$ is called right-dense, right-scattered, left-dense and left-scattered if $\sigma(t)=t, \sigma(t)>t, \rho(t)=t$ and $\rho(t)<t$, respectively. Throughout the text we let $[a, b]=\{t \in \mathbb{T}: a \leq t \leq b\}$ with $a, b \in \mathbb{T}$. We define $\mathbb{T}^{\kappa}=\mathbb{T} \backslash(\rho(b), b]$ and $\mathbb{T}^{\kappa^{2}}=\left(\mathbb{T}^{\kappa}\right)^{\kappa}$. The graininess function $\mu: \mathbb{T} \rightarrow[0, \infty)$ is defined by $\mu(t)=\sigma(t)-t$, for all $t \in \mathbb{T}$. We say that a function $f: \mathbb{T} \rightarrow \mathbb{R}$ is delta differentiable at $t \in \mathbb{T}^{\kappa}$ if there is a number $f^{\Delta}(t)$ such that for all $\varepsilon>0$ there exists a neighborhood $U$ of $t$ (i.e., $U=(t-\delta, t+\delta) \cap \mathbb{T}$ for some $\delta>0)$ such that

$$
\left|f(\sigma(t))-f(s)-f^{\Delta}(t)(\sigma(t)-s)\right| \leq \varepsilon|\sigma(t)-s| \text {, for all } s \in U .
$$

We call $f^{\Delta}(t)$ the delta derivative of $f$ at $t$. For delta differentiable $f$ and $g$, the next formulas hold:

$$
\begin{aligned}
f^{\sigma}(t) & =f(t)+\mu(t) f^{\Delta}(t), \\
(f g)^{\Delta}(t) & =f^{\Delta}(t) g^{\sigma}(t)+f(t) g^{\Delta}(t) \\
& =f^{\Delta}(t) g(t)+f^{\sigma}(t) g^{\Delta}(t),
\end{aligned}
$$

where we abbreviate $f \circ \sigma$ by $f^{\sigma}$. A function $f: \mathbb{T} \rightarrow \mathbb{R}$ is called $r d$-continuous if it is continuous in right-dense points and if its left-sided limit exists in left-dense points. We denote the set of all rd-continuous functions by $\mathrm{C}_{\mathrm{rd}}$ or $\mathrm{C}_{\mathrm{rd}}[\mathbb{T}]$ and the set of all delta differentiable functions with rd-continuous derivative by $\mathrm{C}_{\mathrm{rd}}^{1}$ or $\mathrm{C}_{\mathrm{rd}}^{1}[\mathbb{T}]$. It is useful to provide an example to the reader with the concepts introduced so far. Consider $\mathbb{T}=\bigcup_{k=0}^{\infty}[2 k, 2 k+1]$. For this time scale,

$$
\mu(t)= \begin{cases}0 & \text { if } t \in \bigcup_{k=0}^{\infty}[2 k, 2 k+1) ; \\ 1 & \text { if } t \in \bigcup_{k=0}^{\infty}\{2 k+1\} .\end{cases}
$$

Let us consider $t \in[0,1] \cap \mathbb{T}$. Then, we have (see [8, Theorem 1.16])

$$
f^{\Delta}(t)=\lim _{s \rightarrow t} \frac{f(t)-f(s)}{t-s}, t \in[0,1),
$$

provided this limit exists, and

$$
f^{\Delta}(1)=\frac{f(2)-f(1)}{2-1}
$$

provided $f$ is continuous at $t=1$. Let

$$
f(t)= \begin{cases}t & \text { if } t \in[0,1) \\ 2 & \text { if } t=1\end{cases}
$$

We observe that at $t=1 f$ is rd-continuous ( since $\lim _{t \rightarrow 1} f(t)=1$ ) but not continuous (since $f(1)=2$ ). 
It is known that rd-continuous functions possess an antiderivative, i.e., there exists a function $F$ with $F^{\Delta}=f$, and in this case an integral is defined by $\int_{a}^{b} f(t) \Delta t=F(b)-F(a)$. It satisfies

$$
\int_{t}^{\sigma(t)} f(\tau) \Delta \tau=\mu(t) f(t)
$$

Lemma 2.1 gives the integration by parts formulas of the delta integral:

Lemma 2.1 ([8]). If $a, b \in \mathbb{T}$ and $f, g \in C_{r d}^{1}$, then

$$
\begin{aligned}
& \int_{a}^{b} f(\sigma(t)) g^{\Delta}(t) \Delta t=[(f g)(t)]_{t=a}^{t=b}-\int_{a}^{b} f^{\Delta}(t) g(t) \Delta t, \\
& \int_{a}^{b} f(t) g^{\Delta}(t) \Delta t=[(f g)(t)]_{t=a}^{t=b}-\int_{a}^{b} f^{\Delta}(t) g(\sigma(t)) \Delta t .
\end{aligned}
$$

The following time scale DuBois-Reymond lemma will be useful for our purposes:

Lemma $2.2\left([\underline{6})\right.$. Let $g \in C_{r d}, g:[a, b]^{\kappa} \rightarrow \mathbb{R}^{n}$. Then,

$$
\int_{a}^{b} g^{T}(t) \eta^{\Delta}(t) \Delta t=0, \text { for all } \eta \in C_{r d}^{1} \text { with } \eta(a)=\eta(b)=0
$$

holds if and only if

$$
g(t)=c \text {, on }[a, b]^{\kappa} \text { for some } c \in \mathbb{R}^{n} .
$$

Finally, we prove a simple but useful technical lemma.

Lemma 2.3. Suppose that a continuous function $f: \mathbb{T} \rightarrow \mathbb{R}$ is such that $f^{\sigma}(t)=0$ for all $t \in \mathbb{T}^{\kappa}$. Then, $f(t)=0$ for all $t \in \mathbb{T} \backslash\{a\}$ if $a$ is right-scattered.

Proof. First note that, since $f^{\sigma}(t)=0$, then $f^{\sigma}(t)$ is delta differentiable, hence continuous for all $t \in \mathbb{T}^{\kappa}$. Now, if $t$ is right-dense, the result is obvious. Suppose that $t$ is right-scattered. We will analyze two cases: (i) if $t$ is left-scattered, then $t \neq a$ and by hypothesis $0=f^{\sigma}(\rho(t))=f(t)$; (ii) if $t$ is left-dense, then, by the continuity of $f^{\sigma}$ and $f$ at $t$, we can write

$$
\begin{aligned}
& \forall \varepsilon>0 \exists \delta_{1}>0: \forall s_{1} \in\left(t-\delta_{1}, t\right], \text { we have }\left|f^{\sigma}\left(s_{1}\right)-f^{\sigma}(t)\right|<\varepsilon, \\
& \forall \varepsilon>0 \exists \delta_{2}>0: \forall s_{2} \in\left(t-\delta_{2}, t\right], \text { we have }\left|f\left(s_{2}\right)-f(t)\right|<\varepsilon,
\end{aligned}
$$

respectively. Let $\delta=\min \left\{\delta_{1}, \delta_{2}\right\}$ and take $s_{1} \in(t-\delta, t)$. As $\sigma\left(s_{1}\right) \in(t-\delta, t)$, take $s_{2}=\sigma\left(s_{1}\right)$. By (2.5) and (2.6), we have:

$\left|-f^{\sigma}(t)+f(t)\right|=\left|f^{\sigma}\left(s_{1}\right)-f^{\sigma}(t)+f(t)-f\left(s_{2}\right)\right| \leq\left|f^{\sigma}\left(s_{1}\right)-f^{\sigma}(t)\right|+\left|f\left(s_{2}\right)-f(t)\right|<2 \varepsilon$.

Since $\varepsilon$ is arbitrary, $\left|-f^{\sigma}(t)+f(t)\right|=0$, which is equivalent to $f(t)=f^{\sigma}(t)$.

\section{Main results}

We start in 3.1 by defining the isoperimetric problem on time scales and proving a correspondent first-order necessary optimality condition (Theorem 3.4). Then, in 3.2 we show that certain eigenvalue problems can be recast as an isoperimetric problem (Theorem 3.7). 
3.1. Isoperimetric problems. Let $J: \mathrm{C}_{\mathrm{rd}}^{1} \rightarrow \mathbb{R}$ be a functional defined on the function space $\left(\mathrm{C}_{\mathrm{rd}}^{1},\|\cdot\|\right)$ and let $S \subseteq \mathrm{C}_{\mathrm{rd}}^{1}$.

Definition 3.1. The functional $J$ is said to have a local minimum in $S$ at $y_{*} \in S$ if there exists a $\delta>0$ such that $J\left(y_{*}\right) \leq J(y)$ for all $y \in S$ satisfying $\left\|y-y_{*}\right\|<\delta$.

Now, let $J: \mathrm{C}_{\text {rd }}^{1} \rightarrow \mathbb{R}$ be a functional of the form

$$
J(y)=\int_{a}^{b} L\left(t, y^{\sigma}(t), y^{\Delta}(t)\right) \Delta t,
$$

where $L(t, x, v):[a, b]^{\kappa} \times \mathbb{R} \times \mathbb{R} \rightarrow \mathbb{R}$ has continuous partial derivatives $L_{x}(t, x, v)$ and $L_{v}(t, x, v)$, respectively with respect to the second and third variables, for all $t \in$ $[a, b]^{\kappa}$, and is such that $L\left(t, y^{\sigma}(t), y^{\Delta}(t)\right), L_{x}\left(t, y^{\sigma}(t), y^{\Delta}(t)\right)$ and $L_{v}\left(t, y^{\sigma}(t), y^{\Delta}(t)\right)$ are rd-continuous in $t$ for all $y \in \mathrm{C}_{\mathrm{rd}}^{1}$. The isoperimetric problem consists of finding functions $y$ satisfying given boundary conditions

$$
y(a)=y_{a}, y(b)=y_{b},
$$

and a constraint of the form

$$
I(y)=\int_{a}^{b} g\left(t, y^{\sigma}(t), y^{\Delta}(t)\right) \Delta t=l,
$$

where $g(t, x, v):[a, b]^{\kappa} \times \mathbb{R} \times \mathbb{R} \rightarrow \mathbb{R}$ has continuous partial derivatives with respect to the second and third variables for all $t \in[a, b]^{\kappa}, g\left(t, y^{\sigma}(t), y^{\Delta}(t)\right)$, $g_{x}\left(t, y^{\sigma}(t), y^{\Delta}(t)\right)$ and $g_{v}\left(t, y^{\sigma}(t), y^{\Delta}(t)\right)$ are rd-continuous in $t$ for all $y \in \mathrm{C}_{\mathrm{rd}}^{1}$, and $l$ is a specified real constant, that takes (3.1) to a minimum.

Definition 3.2. We say that a function $y \in \mathrm{C}_{\mathrm{rd}}^{1}$ is admissible for the isoperimetric problem if it satisfies (3.2) and (3.3).

Definition 3.3. An admissible function $y_{*}$ is said to be an extremal for $I$ if it satisfies the following equation (cf. [6]):

$$
g_{v}\left(t, y_{*}^{\sigma}(t), y_{*}^{\Delta}(t)\right)-\int_{a}^{t} g_{x}\left(\tau, y_{*}^{\sigma}(\tau), y_{*}^{\Delta}(\tau)\right) \Delta \tau=c
$$

for all $t \in[a, b]^{\kappa}$ and some constant $c$.

Theorem 3.4. Suppose that $J$ has a local minimum at $y_{*} \in C_{r d}^{1}$ subject to the boundary conditions (3.2) and the isoperimetric constraint (3.3), and that $y_{*}$ is not an extremal for the functional $I$. Then, there exists a Lagrange multiplier constant $\lambda$ such that $y_{*}$ satisfies the following equation:

$$
F_{v}^{\Delta}\left(t, y_{*}^{\sigma}(t), y_{*}^{\Delta}(t)\right)-F_{x}\left(t, y_{*}^{\sigma}(t), y_{*}^{\Delta}(t)\right)=0, \text { for all } t \in[a, b]^{\kappa^{2}},
$$

where $F=L-\lambda g$ and $F_{v}^{\Delta}$ denotes the delta derivative of a composition.

Proof. Let $y_{*}$ be a local minimum for $J$ and consider neighboring functions of the form

$$
\hat{y}=y_{*}+\varepsilon_{1} \eta_{1}+\varepsilon_{2} \eta_{2},
$$

where for each $i \in\{1,2\}, \varepsilon_{i}$ is a sufficiently small parameter $\left(\varepsilon_{1}\right.$ and $\varepsilon_{2}$ must be such that $\left\|\hat{y}-y^{*}\right\|<\delta$, for some $\delta>0-$ see Definition [3.1), $\eta_{i}(x) \in \mathrm{C}_{\mathrm{rd}}^{1}$ and $\eta_{i}(a)=\eta_{i}(b)=0$. Here, $\eta_{1}$ is an arbitrary fixed function and $\eta_{2}$ is a fixed function that we will choose later. 
First we show that (3.5) has a subset of admissible functions for the isoperimetric problem. Consider the quantity

$$
I(\hat{y})=\int_{a}^{b} g\left(t, y_{*}^{\sigma}(t)+\varepsilon_{1} \eta_{1}^{\sigma}(t)+\varepsilon_{2} \eta_{2}^{\sigma}(t), y_{*}^{\Delta}(t)+\varepsilon_{1} \eta_{1}^{\Delta}(t)+\varepsilon_{2} \eta_{2}^{\Delta}(t)\right) \Delta t .
$$

Then we can regard $I(\hat{y})$ as a function of $\varepsilon_{1}$ and $\varepsilon_{2}$, say $I(\hat{y})=\hat{Q}\left(\varepsilon_{1}, \varepsilon_{2}\right)$. Since $y_{*}$ is a local minimum for $J$ subject to the boundary conditions and the isoperimetric constraint, putting $Q\left(\varepsilon_{1}, \varepsilon_{2}\right)=\hat{Q}\left(\varepsilon_{1}, \varepsilon_{2}\right)-l$ we have that

$$
Q(0,0)=0 .
$$

By the conditions imposed on $g$, we have

$$
\begin{aligned}
\frac{\partial Q}{\partial \varepsilon_{2}}(0,0) & =\int_{a}^{b}\left[g_{x}\left(t, y_{*}^{\sigma}(t), y_{*}^{\Delta}(t)\right) \eta_{2}^{\sigma}(t)+g_{v}\left(t, y_{*}^{\sigma}(t), y_{*}^{\Delta}(t)\right) \eta_{2}^{\Delta}(t)\right] \Delta t \\
& =\int_{a}^{b}\left[g_{v}\left(t, y_{*}^{\sigma}(t), y_{*}^{\Delta}(t)\right)-\int_{a}^{t} g_{x}\left(\tau, y_{*}^{\sigma}(\tau), y_{*}^{\Delta}(\tau)\right) \Delta \tau\right] \eta_{2}^{\Delta}(t) \Delta t
\end{aligned}
$$

where (3.7) follows from (2.3) and the fact that $\eta_{2}(a)=\eta_{2}(b)=0$. Now, the function

$$
E(t)=g_{v}\left(t, y_{*}^{\sigma}(t), y_{*}^{\Delta}(t)\right)-\int_{a}^{t} g_{x}\left(\tau, y_{*}^{\sigma}(\tau), y_{*}^{\Delta}(\tau)\right) \Delta \tau
$$

is rd-continuous on $[a, b]^{\kappa}$. Hence, we can apply Lemma 2.2 to show that there exists a function $\eta_{2} \in \mathrm{C}_{\mathrm{rd}}^{1}$ such that

$$
\int_{a}^{b}\left[g_{v}\left(t, y_{*}^{\sigma}(t), y_{*}^{\Delta}(t)\right)-\int_{a}^{t} g_{x}\left(\tau, y_{*}^{\sigma}(\tau), y_{*}^{\Delta}(\tau)\right) \Delta \tau\right] \eta_{2}^{\Delta}(t) \Delta t \neq 0,
$$

provided $y_{*}$ is not an extremal for $I$, which is indeed the case. We have just proved that

$$
\frac{\partial Q}{\partial \varepsilon_{2}}(0,0) \neq 0
$$

Using (3.6) and (3.8), the implicit function theorem asserts that there exist neighborhoods $N_{1}$ and $N_{2}$ of $0, N_{1} \subseteq\left\{\varepsilon_{1}\right.$ from (3.5) $\} \cap \mathbb{R}$ and $N_{2} \subseteq\left\{\varepsilon_{2}\right.$ from (3.5) $\} \cap \mathbb{R}$, and a function $\varepsilon_{2}: N_{1} \rightarrow \mathbb{R}$ such that for all $\varepsilon_{1} \in N_{1}$ we have

$$
Q\left(\varepsilon_{1}, \varepsilon_{2}\left(\varepsilon_{1}\right)\right)=0
$$

which is equivalent to $\hat{Q}\left(\varepsilon_{1}, \varepsilon_{2}\left(\varepsilon_{1}\right)\right)=l$. Now we derive the necessary condition (3.4). Consider the quantity $J(\hat{y})=K\left(\varepsilon_{1}, \varepsilon_{2}\right)$. By hypothesis, $K$ is minimum at $(0,0)$ subject to the constraint $Q(0,0)=0$, and we have proved that $\nabla Q(0,0) \neq \mathbf{0}$. We can appeal to the Lagrange multiplier rule (see, e.g., [15, Theorem 4.1.1]) to assert that there exists a number $\lambda$ such that

$$
\nabla(K(0,0)-\lambda Q(0,0))=\mathbf{0} .
$$

Having in mind that $\eta_{1}(a)=\eta_{1}(b)=0$, we can write:

$$
\begin{aligned}
\frac{\partial K}{\partial \varepsilon_{1}}(0,0) & =\int_{a}^{b}\left[L_{x}\left(t, y_{*}^{\sigma}(t), y_{*}^{\Delta}(t)\right) \eta_{1}^{\sigma}(t)+L_{v}\left(t, y_{*}^{\sigma}(t), y_{*}^{\Delta}(t)\right) \eta_{1}^{\Delta}(t)\right] \Delta t \\
& =\int_{a}^{b}\left[L_{v}\left(t, y_{*}^{\sigma}(t), y_{*}^{\Delta}(t)\right)-\int_{a}^{t} L_{x}\left(\tau, y_{*}^{\sigma}(\tau), y_{*}^{\Delta}(\tau)\right) \Delta \tau\right] \eta_{1}^{\Delta}(t) \Delta t .
\end{aligned}
$$


Similarly, we have that

$$
\frac{\partial Q}{\partial \varepsilon_{1}}(0,0)=\int_{a}^{b}\left[g_{v}\left(t, y_{*}^{\sigma}(t), y_{*}^{\Delta}(t)\right)-\int_{a}^{t} g_{x}\left(\tau, y_{*}^{\sigma}(\tau), y_{*}^{\Delta}(\tau)\right) \Delta \tau\right] \eta_{1}^{\Delta}(t) \Delta t
$$

Combining (3.9), (3.10) and (3.11), we obtain

$$
\int_{a}^{b}\left\{L_{v}(\cdot)-\int_{a}^{t} L_{x}(\cdot \cdot) \Delta \tau-\lambda\left(g_{v}(\cdot)-\int_{a}^{t} g_{x}(\cdot \cdot) \Delta \tau\right)\right\} \eta_{1}^{\Delta}(t) \Delta t=0,
$$

where $(\cdot)=\left(t, y_{*}^{\sigma}(t), y_{*}^{\Delta}(t)\right)$ and $(\cdot \cdot)=\left(\tau, y_{*}^{\sigma}(\tau), y_{*}^{\Delta}(\tau)\right)$. Since $\eta_{1}$ is arbitrary, Lemma 2.2 implies that there exists a constant $d$ such that

$$
L_{v}(\cdot)-\lambda g_{v}(\cdot)-\left(\int_{a}^{t}\left[L_{x}(\cdot \cdot)-\lambda g_{x}(\cdot \cdot)\right] \Delta \tau\right)=d, t \in[a, b]^{\kappa},
$$

or

$$
F_{v}(\cdot)-\int_{a}^{t} F_{x}(\cdot \cdot) \Delta \tau=d,
$$

with $F=L-\lambda g$. Since the integral and the constant in (3.12) are delta differentiable, we obtain the desired necessary optimality condition (3.4).

Remark 3.5. Theorem 3.4 remains valid when $y_{*}$ is assumed to be a local maximizer of the isoperimetric problem (3.1)-(3.3).

Example 3.6. Suppose that we want to find functions defined on $[-a, a] \cap \mathbb{T}$ that take

$$
J(y)=\int_{-a}^{a} y^{\sigma}(t) \Delta t
$$

to its largest value (see Remark 3.5) and that satisfy the conditions

$$
y(-a)=y(a)=0, \quad I(y)=\int_{-a}^{a} \sqrt{1+\left(y^{\Delta}(t)\right)^{2}} \Delta t=l>2 a .
$$

Note that if $y$ is an extremal for $I$, then $y$ is a line segment [6], and therefore $y(t)=0$ for all $t \in[-a, a]$. This implies that $I(y)=2 a>2 a$, which is a contradiction. Hence, $I$ has no extremals satisfying the boundary conditions and the isoperimetric constraint. Using Theorem 3.4, let

$$
F=L-\lambda g=y^{\sigma}-\lambda \sqrt{1+\left(y^{\Delta}\right)^{2}} .
$$

Because

$$
F_{x}=1, \quad F_{v}=\lambda \frac{y^{\Delta}}{\sqrt{1+\left(y^{\Delta}\right)^{2}}},
$$

a necessary optimality condition is given by the following delta-differential equation:

$$
\lambda\left(\frac{y^{\Delta}}{\sqrt{1+\left(y^{\Delta}\right)^{2}}}\right)^{\Delta}-1=0, \quad t \in[-a, a]^{\kappa^{2}} .
$$

The reader interested in the study of delta-differential equations on time scales is referred to $\mathbf{9}$ and references therein. 
If we restrict ourselves to times scales $\mathbb{T}$ with $\sigma(t)=a_{1} t+a_{0}$ for some $a_{1} \in$ $\mathbb{R}^{+}$and $a_{0} \in \mathbb{R}\left(a_{0}=0\right.$ and $a_{1}=1$ for $\mathbb{T}=\mathbb{R} ; a_{0}=a_{1}=1$ for $\left.\mathbb{T}=\mathbb{Z}\right)$, it follows from the results in $1 \mathbf{1 0}$ that the same proof of Theorem 3.4 can be used, mutatis mutandis, to obtain a necessary optimality condition for the higher-order isoperimetric problem (i.e., when $L$ and $g$ contain higher order delta derivatives). In this case, the necessary optimality condition (3.4) is generalized to

$$
\sum_{i=0}^{r}(-1)^{i}\left(\frac{1}{a_{1}}\right)^{\frac{(i-1) i}{2}} F_{u_{i}}^{\Delta^{i}}\left(t, y_{*}^{\sigma^{r}}(t), y_{*}^{\sigma^{r-1} \Delta}(t), \ldots, y_{*}^{\sigma \Delta^{r-1}}(t), y_{*}^{\Delta^{r}}(t)\right)=0,
$$

where $F=L-\lambda g$, and functions $\left(t, u_{0}, u_{1}, \ldots, u_{r}\right) \rightarrow L\left(t, u_{0}, u_{1}, \ldots, u_{r}\right)$ and $\left(t, u_{0}, u_{1}, \ldots, u_{r}\right) \rightarrow g\left(t, u_{0}, u_{1}, \ldots, u_{r}\right)$ are assumed to have (standard) partial derivatives with respect to $u_{0}, \ldots, u_{r}, r \geq 1$, and partial delta derivative with respect to $t$ of order $r+1$.

3.2. Sturm-Liouville eigenvalue problems. Eigenvalue problems on time scales have been studied in [2]. Consider the following Sturm-Liouville eigenvalue problem: find nontrivial solutions to the delta-differential equation

$$
y^{\Delta^{2}}(t)+q(t) y^{\sigma}(t)+\lambda y^{\sigma}(t)=0, t \in[a, b]^{\kappa^{2}},
$$

for the unknown $y:[a, b] \rightarrow \mathbb{R}$ subject to the boundary conditions

$$
y(a)=y(b)=0 .
$$

Here $q:[a, b] \rightarrow \mathbb{R}$ is a continuous function and $y^{\Delta^{2}}=\left(y^{\Delta}\right)^{\Delta}$.

Generically, the only solution to equation (3.13) that satisfies the boundary conditions (3.14) is the trivial solution, $y(t)=0$ for all $t \in[a, b]$. There are, however, certain values of $\lambda$ that lead to nontrivial solutions. These are called eigenvalues and the corresponding nontrivial solutions are called eigenfunctions. These eigenvalues may be arranged as $-\infty<\lambda_{1}<\lambda_{2}<\ldots$ (see Theorem 1 of [2]) and $\lambda_{1}$ is called the first eigenvalue.

Consider the functional defined by

$$
J(y)=\int_{a}^{b}\left(\left(y^{\Delta}\right)^{2}(t)-q(t)\left(y^{\sigma}\right)^{2}(t)\right) \Delta t,
$$

and suppose that $y_{*} \in \mathrm{C}_{\text {rd }}^{2}$ (functions that are twice delta differentiable with rdcontinuous second delta derivative) is a local minimum for $J$ subject to the boundary conditions (3.14) and the isoperimetric constraint

$$
I(y)=\int_{a}^{b}\left(y^{\sigma}\right)^{2}(t) \Delta t=1 .
$$

If $y_{*}$ is an extremal for $I$, then we would have $-2 y^{\sigma}(t)=0, t \in[a, b]^{\kappa}$. Noting that $y(a)=0$, using Lemma 2.3 we would conclude that $y(t)=0$ for all $t \in[a, b]$. No extremals for $I$ can therefore satisfy the isoperimetric condition (3.16). Hence, by Theorem 3.4 there is a constant $\lambda$ such that $y_{*}$ satisfies

$$
F_{y^{\Delta}}^{\Delta}\left(t, y_{*}^{\sigma}(t), y_{*}^{\Delta}(t)\right)-F_{y^{\sigma}}\left(t, y_{*}^{\sigma}(t), y_{*}^{\Delta}(t)\right)=0,
$$

with $F=\left(y^{\Delta}\right)^{2}-q\left(y^{\sigma}\right)^{2}-\lambda\left(y^{\sigma}\right)^{2}$. It is easily seen that (3.17) is equivalent to (3.13). The isoperimetric problem thus corresponds to the Sturm-Liouville problem augmented by the normalizing condition (3.16), which simply scales the eigenfunctions. Here, the Lagrange multiplier plays the role of the eigenvalue. 
Theorem 3.7. Let $\lambda_{1}$ be the first eigenvalue for the Sturm-Liouville problem (3.13) with boundary conditions (3.14), and let $y_{1}$ be the corresponding eigenfunction normalized to satisfy the isoperimetric constraint (3.16). Then, among functions in $C_{r d}^{2}$ that satisfy the boundary conditions (3.14) and the isoperimetric condition (3.16), the functional $J$ defined by (3.15) has a minimum at $y_{1}$. Moreover, $J\left(y_{1}\right)=\lambda_{1}$.

Proof. Suppose that $J$ has a minimum at $y$ satisfying conditions (3.14) and (3.16). Then $y$ satisfies equation (3.13) and multiplying this equation by $y^{\sigma}$ and delta integrating from $a$ to $b$, we obtain

$$
\int_{a}^{b} y^{\sigma}(t) y^{\Delta^{2}}(t) \Delta t+\int_{a}^{b} q(t)\left(y^{\sigma}\right)^{2}(t) \Delta t+\lambda \int_{a}^{b}\left(y^{\sigma}\right)^{2}(t) \Delta t=0
$$

Since $y(a)=y(b)=0$,

$$
\int_{a}^{b} y^{\sigma}(t) y^{\Delta^{2}}(t) \Delta t=\left[y(t) y^{\Delta}(t)\right]_{t=a}^{t=b}-\int_{a}^{b}\left(y^{\Delta}\right)^{2} \Delta t=-\int_{a}^{b}\left(y^{\Delta}\right)^{2} \Delta t,
$$

and by (3.16), (3.18) reduces to

$$
\int_{a}^{b}\left[\left(y^{\Delta}\right)^{2}-q(t)\left(y^{\sigma}\right)^{2}(t)\right] \Delta t=\lambda
$$

that is, $J(y)=\lambda$. Due to the isoperimetric condition, $y$ must be a nontrivial solution to (3.13) and therefore $\lambda$ must be an eigenvalue. Since there exists a least element within the eigenvalues, $\lambda_{1}$, and a corresponding eigenfunction $y_{1}$ normalized to meet the isoperimetric condition, the minimum value for $J$ is $\lambda_{1}$ and $J\left(y_{1}\right)=\lambda_{1}$.

\section{References}

[1] R. Agarwal, M. Bohner, D. O'Regan and A. Peterson, Dynamic equations on time scales: a survey, J. Comput. Appl. Math. 141 (2002), no. 1-2, 1-26.

[2] R. Agarwal, M. Bohner and P. J. Y. Wong, Sturm-Liouville eigenvalue problems on time scales, Appl. Math. Comput. 99 (1999), no. 2-3, 153-166.

[3] C. D. Ahlbrandt and B. J. Harmsen, Discrete versions of continuous isoperimetric problems, J. Differ. Equations Appl. 3 (1998), no. 5-6, 449-462.

[4] F. M. Atici, D. C. Biles and A. Lebedinsky, An application of time scales to economics, Math. Comput. Modelling 43 (2006), no. 7-8, 718-726.

[5] Z. Bartosiewicz and D. F. M. Torres, Noether's theorem on time scales, J. Math. Anal. Appl. 342 (2008), no. 2, 1220-1226.

[6] M. Bohner, Calculus of variations on time scales, Dynam. Systems Appl. 13 (2004), no. 3-4, 339-349.

[7] M. Bohner and G. Sh. Guseinov, Double integral calculus of variations on time scales, Comput. Math. Appl. 54 (2007), no. 1, 45-57.

[8] M. Bohner and A. Peterson, Dynamic equations on time scales, Birkhäuser Boston, Boston, MA, 2001.

[9] M. Bohner and A. Peterson, Advances in dynamic equations on time scales, Birkhäuser Boston, Boston, MA, 2003.

[10] R. A. C. Ferreira and D. F. M. Torres, Higher-order calculus of variations on time scales, Proceedings of the Workshop on Mathematical Control Theory and Finance, Lisbon, 10-14 April 2007, 150-158. In: Mathematical Control Theory and Finance, Sarychev, A.; Shiryaev, A.; Guerra, M.; Grossinho, M.d.R. (Eds.), Springer, 2008.

[11] R. A. C. Ferreira and D. F. M. Torres, Remarks on the calculus of variations on time scales, Int. J. Ecol. Econ. Stat. 9 (2007), no. F07, 65-73.

[12] S. Hilger, Analysis on measure chains - a unified approach to continuous and discrete calculus, Results Math. 18 (1990), no. 1-2, 18-56.

[13] S. Hilger, Differential and difference calculus-unified!, Nonlinear Anal. 30 (1997), no. 5, 2683-2694. 
[14] R. Hilscher and V. Zeidan, Calculus of variations on time scales: weak local piecewise $C_{\text {rd }}^{1}$ solutions with variable endpoints, J. Math. Anal. Appl. 289 (2004), no. 1, 143-166.

[15] B. van Brunt, The calculus of variations, Springer, New York, 2004.

Department of Mathematics, University of Aveiro, 3810-193 Aveiro, Portugal

E-mail address: ruiacferreira@ua.pt

Department of Mathematics, University of Aveiro, 3810-193 Aveiro, Portugal

E-mail address: delfim@ua.pt 